\title{
Tritylamine (triphenylmethylamine) in organic synthesis; II. The reaction of tritylamine with oxiranes - synthesis of $\mathrm{N}$-trityl- $\beta$ - aminoalcohols
}

\author{
Mirosław Soroka* and Waldemar Goldeman \\ Politechnika Wrocławska, Instytut Chemii Organicznej, Biochemii i Biotechnologii, Wybrzeże \\ Wyspiańskiego 27, 50370 Wrocław, Poland \\ E-mail: soroka@kchf.ch.pwr.wroc.pl
}

\section{Dedicated to Prof. Henry J. Shine on the occasion of his $80^{\text {th }}$ birthday anniversary and for his outstanding contribution to organic chemistry, perfect lecturing and excellence in teaching}

(received 09 May 03; accepted 19 June 03; published on the web 21 June 03)

\begin{abstract}
The tritylamine (triphenylmethylamine) reacts regioselectively with oxiranes to give crystalline $\mathrm{N}$-tritylated $\beta$-aminoalcohols - useful building blocks in organic synthesis, with reasonable yield. The N-trityl- $\beta$-aminoalcohols hydrolyze quantitatively to $\beta$-aminoalcohol hydrochlorides by means of hydrochloric acid.
\end{abstract}

Keywords: Tritylamine, ammonia equivalent, oxirane, epoxide ring opening, $\beta$-aminoalcohol hydrochlorides

\section{Introduction}

The ring-opening reaction of oxiranes with ammonia is one of the oldest reactions in history of organic chemistry. For the first time it was described in 1859 by Würtz. ${ }^{1}$ To date, this reaction was repeated countlessly, reinvestigated, optimized, etc., by several authors. ${ }^{2}$ It was also a subject of numerous patents since its application in industry for manufacturing of the aminoalcohols - ingredients of many daily used products. ${ }^{3}$ There are some limitations of this reaction. ${ }^{2}$ The main problems are the low regio- and chemoselectivity, which result always in a complex mixture of products. For example, in the reaction of oxirane with ammonia, even with large excess of ammonia, there are three main products: 2-aminoethanol, diethanoloamine and triethanoloamine. ${ }^{2}$ Separation of such a mixture, usually by vacuum distillation, is a tedious and costly task. A similar reaction of ammonia with substituted oxiranes show additional problems with regioselectivity, so far not very well understood. The reaction of ammonia with oxirane is 
also highly exothermic, therefore difficult to control which is the reason of many catastrophes in industry. ${ }^{2,3}$

The reactions of ammonolysis and aminolysis of oxiranes are a classical route to aminoalcohols - important building blocks and chiral auxiliaries in organic synthesis, mainly for medicinal chemistry, since many pharmaceuticals contain the O-C-C-N moiety. Sometimes the orthogonally protected $\beta$-aminoalcohol is needed for introduction of a $\beta$-aminoalcohol moiety into a target molecule. $\mathrm{N}$-tritylated $\beta$-aminoalcohols are reagents of choice for this purpose. ${ }^{4}$

Years ago we introduced successfully tritylamine as an ammonia equivalent in the Mannich type reaction for the synthesis of N-tritylalkanimines and N-trityl-1-aminoalkylphosphonates. ${ }^{5}$ We assumed that a highly stereoelectronic hindered trityl group should moderate the reactivity of the amino function, therefore, it should improve regioselectivity and decrease the rate of the ring-opening reaction of epoxides. Moreover, the reaction of epoxides with tritylamine seems to be the easiest way for preparation of $\mathrm{N}$-tritylated $\beta$-aminoalcohols (Scheme 1).<smiles>[R]C(O)CNCCCCCCCCC(O)CNC(c1ccccc1)(c1ccccc1)c1ccccc1</smiles>

Scheme 1

Despite a few papers devoted to the tritylation of $\beta$-aminoalcohols ${ }^{4}$ we found no examples of the reaction of tritylamine with simple epoxides in the literature. ${ }^{6}$

\section{Results and Discussion}

First, we examined the reaction of tritylamine with ethylene oxide in methanol. ${ }^{7}$ Surprisingly and contrary to the reaction of oxiranes with benzylic ${ }^{8}$ or benzhydrylic ${ }^{9}$ type of amines, the tritylamine reacts with ethylene oxide with difficulty. However, when the reaction mixture was stirred patiently for a long time or kept at elevated temperature, we obtained a good conversion of tritylamine into 2-(triphenylmethylamino)ethanol. The crude reaction mixture (essayed by NMR) contains almost quantitatively the desired product. Simple evaporation of the reaction mixture leaves practically pure 2-(triphenylmethylamino)ethanol ${ }^{10}$ which could be recrystallized $^{11}$ or converted directly to its derivatives or deblocked to 2-aminoethanol hydrochloride by short reflux with hydrochloric acid.

Also methyloxirane or ethyloxirane gave good yields of $\mathrm{N}$-tritylated $\beta$-aminoalcohols with, what is worth noticing, very high regioselectivity. ${ }^{12}$ We did not observe any detectable amount of the second regioisomer by means of proton NMR. Also in these cases a short reflux with hydrochloric acid gave suitable hydrochlorides of the 1-aminoalkan-2-ols. An equimolar mixture of phenyloxirane (styrene oxide) and tritylamine in methanol heated on a boiling water bath for two days, gave exclusively 1-phenyl-2-(triphenylmethyl)ethanol, without 2-phenyl-1(triphenylmethylamino)ethanol ${ }^{13}$ with an average yield. The hydrolysis of a recrystallized product with $2 \mathrm{M}$ hydrochloric acid ${ }^{14}$ gave 2-amino-1-phenylethanol hydrochloride. The 
epichlorohydrin reacted with tritylamine not only regio- but also chemoselectively. In the reaction mixture we found only ring opening product and only in $\beta$-position. However, in this case the reaction mixture was very difficult to purify because of cocrystallization of the product with unreacted tritylamine. However, when the crude product (contained about $60 \%$ of 3-chloro1-(triphenylmethylamino)propan-2-ol and 40\% of tritylamine) was refluxed with hydrochloric acid we obtained exclusively 1-amino-3-chloropropan-2-ol hydrochloride contaminated only by ammonium chloride - the byproduct from the hydrolysis of unreacted tritylamine. Repeating the same reaction in 1/5 molar ratio gave an oily product containing 92\% of 3-chloro-1(triphenylmethylamino)propan-2-ol. This reaction is currently under investigation for its potential applications for the synthesis of azetidin-3-ol derivatives. ${ }^{15}$

The results are summarized in table 1.

Table 1. Results of the ring-opening reactions of oxiranes by tritylamine

\begin{tabular}{ccccc}
\hline $\mathrm{R}$ & $\begin{array}{c}\text { tritylamine/oxirane } \\
\text { molar ratio }\end{array}$ & $\begin{array}{c}\text { Reaction time } \\
\text { hours }\end{array}$ & $\begin{array}{c}\text { Temperature } \\
{ }^{\circ} \mathrm{C}\end{array}$ & $\begin{array}{c}\text { Yield } \\
\text { (nmr/isolated) } \\
\%\end{array}$ \\
\hline $\mathrm{H}$ & $1 / 10$ & 2 & 80 & $-/ 99$ \\
$\mathrm{Me}$ & $1 / 10$ & 8 & 80 & $-/ 97$ \\
$\mathrm{Et}$ & $1 / 10$ & 12 & 100 & $-/ 89$ \\
$\mathrm{Ph}$ & $1 / 1.1$ & 18 & 70 & $41 /-$ \\
$\mathrm{Ph}$ & $1 / 5$ & 32 & 100 & $50 / 40$ \\
$\mathrm{ClCH}_{2}$ & $1 / 1 / 1$ & 18 & 75 & $60 /-$ \\
$\mathrm{ClCH}_{2}$ & $1 / 5$ & 15 & 60 & $92 /$ oily \\
\hline
\end{tabular}

\section{Conclusions}

Tritylamine reacts with epoxides in methanol at elevated temperature to give the corresponding $\mathrm{N}$-tritylated $\beta$-aminoalcohols with reasonable yields and perfect regioselectivity. The $\mathrm{N}$-tritylated $\beta$-aminoalcohols refluxed with hydrochloric acid give quantitatively $\beta$-aminoalcohol hydrochlorides and tritanol as a side product. The tritanol could be easily separated from the reaction mixture and then converted to tritylamine, which can be recycled. Therefore, the trityl group mimicks an "ammonia transferase" - which delivers the ammonia as an amino moiety to a specific place in the target molecule.

\section{Experimental Section}

General Procedures. NMR spectra were recorded on a Bruker Avance $300 \mathrm{MHz}$ spectrometer locked on deuterium. Chemical shifts $(\delta[\mathrm{ppm}])$ were calculated from chemical shift of deuterium lock and were not calibrated. FTIR spectra were measured on Perkin Elmer 2000 spectrometer in $\mathrm{KBr}$ pellets $(1 / 200)$ by Mrs. Elżbieta Mróź and the elemental analyses by 
combustion were done by Mrs. Czesława Andrzejewska (both from our institute). Melting points were determined on the Boetius microscope with electrical hot plate and were corrected. Since all samples decomposed when heated and melted, the melting points in these cases depended on the rate of heating and so they are not "physical constants". The reactions were performed in the pressure tube reactor equipped with Teflon ${ }^{\circledR}$ lined steel valve (Lab Glass Inc.) and magnetic stirrer with Teflon $^{\circledR}$ lined stirrer bar. The required epoxides were acquired from local manufacturer. All solvents were of commercial quality and purchased from local supplier (POCh Gliwice). Tritylamine was prepared by ammonolysis of trityl chloride (it could be also purchased from Acros Organics).

\section{A typical procedure}

When the epoxides are easily available and not expensive, the best procedure is to use an excess of epoxide, since tritylated aminoalcohols do not react with them. Alternatively, an excess of tritylamine could be applied since the ammonium salt is the sole side product after the final deblocking procedure. To a magnetically stirred cold suspension of tritylamine (5.2 g, $20 \mathrm{mmol})$ in methanol $(50 \mathrm{~mL})$ placed in a pressure tube reactor, precooled epoxide (about $0,2 \mathrm{~mol}$ ) was slowly added. The reaction mixture was stirred to reach the temperature about $25^{\circ} \mathrm{C}$, then slowly heated on a water bath to $80^{\circ} \mathrm{C}$, then it was stirred for the time and temperature indicated in Table 1. After reaction, the mixture was cooled to room temperature, evaporated to dryness under reduced pressure and the residue assayed by means of NMR. The residue was treated with hexane $(10 \mathrm{~mL})$ and left in the refrigerator for crystallization. Crystalline material was filtered, washed with cold hexane $(2 \times 5 \mathrm{~mL})$ and dried in a vacuum dessicator. An analytical sample was additionally recrystallized from hexane or dichloromethane/hexane.

2-(Triphenylmethylamino)ethanol. $\mathrm{mp} 70-2^{\circ} \mathrm{C}\left(\right.$ lit. $\left.75-6^{4 b}\right) .{ }^{1} \mathrm{H}$ NMR $\left(\mathrm{CDCl}_{3}\right): 7.44(\mathrm{~d}, 6 \mathrm{H}, \mathrm{o}-$ ArH, $\mathrm{J}=7.4), 7.26(\mathrm{dd}, 6 \mathrm{H}, \mathrm{m}-\mathrm{ArH}, \mathrm{J}=7.1, \mathrm{~J}=7.4), 7.16(\mathrm{t}, 3 \mathrm{H}, \mathrm{p}-\mathrm{ArH}, \mathrm{J}=7.1), 3.65(\mathrm{t}, 2 \mathrm{H}$, $\left.\mathrm{CH}_{2}-\mathrm{O}, \mathrm{J}=5.2\right), 2.33\left(\mathrm{t}, 2 \mathrm{H}, \mathrm{CH}_{2}-\mathrm{N}, \mathrm{J}=5.2\right), 1.8(\mathrm{~s}$, broad, $2 \mathrm{H}, \mathrm{NH}+\mathrm{OH})$. FTIR $(\mathrm{KBr}, 1 / 200)$ : $3357(\mathrm{NH}), 3287$ broad $(\mathrm{OH}), 3055,3018,2922,2868,1595,1489,1446,1178,1117,1069$, $1026,898,764,754,704,642,628,517 \mathrm{~cm}^{-1}$. Anal. Calcd. for $\mathrm{C}_{21} \mathrm{H}_{21} \mathrm{NO}$ : C, 83.1; H, 6.98; N, 4.62. Found: C, 81.1; H, 6.96; N, 4.04.

1-(Triphenylmethylamino)propan-2-ol. mp $103-5^{\circ} \mathrm{C} .{ }^{1} \mathrm{H}$ NMR $\left(\mathrm{CDCl}_{3}\right): 7.43$ (d, 6H, o- $\mathrm{ArH}, \mathrm{J}$ $=7.9$ ), $7.26(\mathrm{dd}, 6 \mathrm{H}, \mathrm{m}-\mathrm{ArH}, \mathrm{J}=7.1, \mathrm{~J}=7.9$ ), 7.17 (t, p-ArH, $\mathrm{J}=7.1), 3.78$ (ddq, $1 \mathrm{H}, \mathrm{CH}-\mathrm{O}, \mathrm{J}=$ $6.2, \mathrm{~J}=6.0, \mathrm{~J}=4.0), 2.7$ (s, broad, $1 \mathrm{H}, \mathrm{NH}), 2.16\left(\mathrm{dd}, 1 \mathrm{H}, \mathrm{CH}_{2}-\mathrm{N}, \mathrm{J}=4,0, \mathrm{~J}=11.0\right), 2.14(\mathrm{dd}, 1 \mathrm{H}$, $\left.\mathrm{CH}_{2}-\mathrm{N}, \mathrm{J}=6,0, \mathrm{~J}=11.0\right), 1.8$ (s, broad, $\left.1 \mathrm{H}, \mathrm{OH}\right), 1.09$ (d, 3H, $\mathrm{CH}_{3}, \mathrm{~J}=6.2$ ). FTIR $(\mathrm{KBr}, 1 / 200)$ : 3421 broad $(\mathrm{OH}), 3329(\mathrm{NH}), 3052,3017,2969,2954,2904,1595,1489,1446,1371,1147$, 1127, 1075, 1023, 921, 896, 767, 753, 709, 699, 644, 625, 538, $491 \mathrm{~cm}^{-1}$. Anal. Calcd. for $\mathrm{C}_{22} \mathrm{H}_{23} \mathrm{NO}: \mathrm{C}, 83.2 ; \mathrm{H}, 7.30 ; \mathrm{N}, 4.41$. Found: $\mathrm{C}, 83.0 ; \mathrm{H}, 7.07 ; \mathrm{N}, 4.17$.

1-(Triphenylmethylamino)butan-2-ol. mp 104-5 ${ }^{\circ} \mathrm{C} .{ }^{1} \mathrm{H}$ NMR $\left(\mathrm{CDCl}_{3}\right): 7.43(\mathrm{~d}, 6 \mathrm{H}, \mathrm{o}-\mathrm{ArH}, \mathrm{J}=$ 7.3), 7.25 (dd, 6H, m-ArH, J = 7.1, J = 7.3), 7.16 (t, 3H, p-ArH, J = 7.1), 3.52 (ddt, 1H, CH-O, J $=7.2, \mathrm{~J}=6.0, \mathrm{~J}=4.1), 2.6(\mathrm{~s}$, broad, $1 \mathrm{H}, \mathrm{NH}), 2.16\left(\mathrm{dd}, 1 \mathrm{H}, \mathrm{CH}_{2}-\mathrm{N}, \mathrm{J}=4.0, \mathrm{~J}=11.0\right), 2.14(\mathrm{dd}, 1 \mathrm{H}$, $\left.\mathrm{CH}_{2}-\mathrm{N}, \mathrm{J}=6.0, \mathrm{~J}=11.0\right), 1.39\left(\mathrm{dq}, 2 \mathrm{H}, \mathrm{CH}_{2}-\mathrm{C}, \mathrm{J}=7.4, \mathrm{~J}=7.2\right), 1.6(\mathrm{~s}$, broad, $1 \mathrm{H}, \mathrm{OH}), 0.85(\mathrm{t}$, $\left.3 \mathrm{H}, \mathrm{CH}_{3}, \mathrm{~J}=7.4\right)$. FTIR $(\mathrm{KBr}, 1 / 200): 3459$ broad $(\mathrm{OH}), 3264(\mathrm{NH}), 3081,3054,3031,3019$, 
2963, 2922, 2863, 1595, 1492, 1447, 1412, 1300, 1239, 1204, 1140, 1102, 1074, 1022, 943, 926, $902,764,745,706,695,646,624,530,479 \mathrm{~cm}^{-1}$. Anal. Calcd. for $\mathrm{C}_{23} \mathrm{H}_{25} \mathrm{NO}$ : C, 83.3; H, 7.60; N, 4.23. Found: C, 83.2; H, 7.86; N, 4.10.

1-Phenyl-2-(triphenylmethylamino)ethanol. The equimolar amounts of phenyloxirane (styrene oxide) and tritylamine were applied in this reaction and after typical procedure the product was recrystallized from hexane/dichloromethane. mp $118-120^{\circ} \mathrm{C} .{ }^{1} \mathrm{H} \mathrm{NMR}\left(\mathrm{CDCl}_{3}\right): 7.43(\mathrm{~d}, 6 \mathrm{H}, \mathrm{o}-$ ArH, J = 7.2), 7.25 (dd, 6H, m-ArH, J = 7.1, J = 7.2), $7.25(\mathrm{~m}, 5 \mathrm{H}, \mathrm{Ph}), 7.15(\mathrm{t}, 3 \mathrm{H}, \mathrm{p}-\mathrm{ArH}, \mathrm{J}=$ 7.1), 4.68 (dd, 1H, CH-O, J = 7.4, J = 4.7), 2.9 (s, broad, 1H, NH), 2.49 (dd, 1H, CH-N, J = 12.3, $\mathrm{J}=7.4), 2.46(\mathrm{dd}, 1 \mathrm{H}, \mathrm{CH}-\mathrm{N}, \mathrm{J}=12.3, \mathrm{~J}=4.7), 1.8(\mathrm{~s}$, broad, 1H, OH). FTIR $(\mathrm{KBr}, 1 / 200): 3460$ broad $(\mathrm{OH}), 3309(\mathrm{NH}), 3055,3025,2960,2901,2850,1595,1490,1447,1415,1340,1309$, $1202,1104,1067,1029,1006,941,903,843,818,765,745,706,696,639,627,549,535,505 \mathrm{~cm}^{-1}$. Anal. Calcd. for $\mathrm{C}_{27} \mathrm{H}_{25} \mathrm{NO}$ : C, 85.5; H, 6.64; N, 3.69. Found: C, 85.5; H, 6.40; N, 3.48.

3-Chloro-1-(triphenylmethylamino)propan-2-ol. A solution of chloromethyloxirane (epichlorohydrin) $4.6 \mathrm{~g}(50 \mathrm{mmol})$ and tritylamine $2.6 \mathrm{~g}(10 \mathrm{mmol})$ in $25 \mathrm{~mL}$ of methanol was refluxed overnight (about $15 \mathrm{~h}$ ). The volatile material was removed by evaporation and oily residue ( $2.9 \mathrm{~g}$ ) was assayed by NMR as a $92 \%$ concentrate of product which contained unreacted tritylamine as a main impurity. ${ }^{1} \mathrm{H}$ NMR $\left(\mathrm{CDCl}_{3}\right)$ : $7.48(\mathrm{~d}, 6 \mathrm{H}, \mathrm{o}-\mathrm{ArH}, \mathrm{J}=7.5), 7.33(\mathrm{dd}, 6 \mathrm{H}, \mathrm{m}-$ $\mathrm{ArH}, \mathrm{J}=7.2, \mathrm{~J}=7.5$ ), 7.29 (s, ArH from unreacted tritylamine), $7.26(\mathrm{t}, 3 \mathrm{H}, \mathrm{p}-\mathrm{ArH}, \mathrm{J}=7.2$ ), 3.94 (dddd, $1 \mathrm{H}, \mathrm{CH}-\mathrm{O}, \mathrm{J}=4.5, \mathrm{~J}=4.8, \mathrm{~J}=6.7, \mathrm{~J}=6.9$ ), 3.69 (dd, 1H, CH-Cl, J = 4.5, J = 11.4), $3.64(\mathrm{dd}, 1 \mathrm{H}, \mathrm{CH}-\mathrm{Cl}, \mathrm{J}=6.7, \mathrm{~J}=11.4), 2.41(\mathrm{dd}, 1 \mathrm{H}, \mathrm{CH}-\mathrm{N}, \mathrm{J}=4.8, \mathrm{~J}=12.3), 2.36$ (dd, 1H, CH$\mathrm{N}, \mathrm{J}=6.9, \mathrm{~J}=12.3$ ), $2.0-2.7$ (s, very broad, $\sim 4 \mathrm{H}, \mathrm{NH}, \mathrm{OH}$ and $\mathrm{NH}_{2}$ from unreacted tritylamine).

\section{Detritylation of $N$-tritylated $\beta$-aminoalcohols}

The detritylation was done only for confirmation of structures and regioselectivity of the reactions of oxiranes with tritylamine. A suspension of N-trityl- $\beta$-aminoalcohol $(0.1 \mathrm{mmol})$ in hydrochloric acid $(6 \mathrm{M}, 10 \mathrm{~mL})$ was refluxed for about $1 \mathrm{~h}$ then cooled to room temperature. The precipitate (triphenylmethanol) was separated by suction, washed with water $(2 \times 3 \mathrm{~mL})$, then the combined filtrates were evaporated and residue was analyzed by means of ${ }^{1} \mathrm{H}$ NMR. In all cases the NMR spectra indicated presence of only one regioisomer of $\beta$-aminoalcohol hydrochlorides.

2-Aminoethanol hydrochloride. ${ }^{1} \mathrm{H}$ NMR $\left(\mathrm{D}_{2} \mathrm{O}\right): 4.71(\mathrm{~s}, \mathrm{HOD}), 3.59\left(\mathrm{t}, 2 \mathrm{H}, \mathrm{CH}_{2}-\mathrm{O}, \mathrm{J}=5.2\right.$ ), $2.91\left(\mathrm{t}, 2 \mathrm{H}, \mathrm{CH}_{2}-\mathrm{N}, \mathrm{J}=5.2\right)$.

1-Aminopropan-2-ol hydrochloride. ${ }^{1} \mathrm{H}$ NMR $\left(\mathrm{D}_{2} \mathrm{O}+\mathrm{D}_{2} \mathrm{SO}_{4}\right)$ : 4.90 (s, HOD), 3.36 (ddq, $1 \mathrm{H}$, $\mathrm{CH}-\mathrm{O}, \mathrm{J}=2.9, \mathrm{~J}=6.4, \mathrm{~J}=9.3$ ), 2.42 (dd, 1H, CH-N, $\mathrm{J}=2.9, \mathrm{~J}=12.8$ ), 2.21 (dd, 1H, CH-N, $\mathrm{J}=$ 9.3, $\mathrm{J}=12.8), 0.54\left(\mathrm{~d}, 3 \mathrm{H}, \mathrm{CH}_{3}, \mathrm{~J}=6.4\right)$.

1-Aminobutan-2-ol hydrochloride. ${ }^{1} \mathrm{H}$ NMR $\left(\mathrm{D}_{2} \mathrm{O}\right): 4.67$ (s, HOD), 3.65 (dddd, $1 \mathrm{H}, \mathrm{CH}-\mathrm{O}, \mathrm{J}=$ 4.2, $\mathrm{J}=4.2, \mathrm{~J}=7.4, \mathrm{~J}=9.9), 3.03(\mathrm{dd}, 1 \mathrm{H}, \mathrm{CH}-\mathrm{N}, \mathrm{J}=4.2, \mathrm{~J}=12.8), 2.76(\mathrm{dd}, 1 \mathrm{H}, \mathrm{CH}-\mathrm{N}, \mathrm{J}=9.9$, $\mathrm{J}=12.8$ ), 1.45 (ddt, $1 \mathrm{H}, \mathrm{CH}-\mathrm{C}, \mathrm{J}=4.2, \mathrm{~J}=7.4, \mathrm{~J}=14.8$ ), $1.43(\mathrm{ddt}, 1 \mathrm{H}, \mathrm{CH}-\mathrm{C}, \mathrm{J}=7.4, \mathrm{~J}=7.4, \mathrm{~J}$ $=14.8), 0.82\left(\mathrm{t}, 3 \mathrm{H}, \mathrm{CH}_{3}, \mathrm{~J}=7.4\right)$. 
2-Amino-1-phenylethanol hydrochloride. ${ }^{1} \mathrm{H}$ NMR $\left(\mathrm{D}_{2} \mathrm{O}+\mathrm{D}_{2} \mathrm{SO}_{4}\right): 6.38(\mathrm{~m}, 5 \mathrm{H}, \mathrm{Ph}), 4.94(\mathrm{~s}$, HOD), 3.96 (dd, 1H, CH-O, J = 3.8, J = 8.9), 2.25 (dd, 1H, CH-N, J = 3.8, J = 12.7), 2.18 (dd, $1 \mathrm{H}, \mathrm{CH}-\mathrm{N}, \mathrm{J}=8.9, \mathrm{~J}=12.7)$.

1-Amino-3-chloropropan-2-ol hydrochloride. ${ }^{1} \mathrm{H}$ NMR $\left(\mathrm{D}_{2} \mathrm{O}\right)$ : 4.71 (s, HOD), 4.02 (dddd, 1H, $\mathrm{CH}-\mathrm{O}, \mathrm{J}=4.1, \mathrm{~J}=4.6, \mathrm{~J}=5.4, \mathrm{~J}=10.0), 3.57$ (dd, 1H, CH-Cl, J = 4.6, J = 11.9), 3.50 (dd, 1H, $\mathrm{CH}-\mathrm{Cl}, \mathrm{J}=5.4, \mathrm{~J}=11.9$ ), $3.09(\mathrm{dd}, 1 \mathrm{H}, \mathrm{CH}-\mathrm{N}, \mathrm{J}=4.1, \mathrm{~J}=12.4), 2.91$ (dd, 1H, CH-N, J = 10.0, J $=12.4$ ), additional three lines at 7.12, 6.94 and 6.77 indicated presence of ammonium chloride derived from hydrolysis of unreacted tritylamine.

\section{References and Notes}

1. (a) Würtz, A. C. R. Hebd. Seances Acad. Sci. 1859, 49, 898. (b) Würtz, A. C. R. Hebd. Seances Acad. Sci. 1861, 53, 338. (c) Würtz, A. Justus Liebigs Ann. Chem. 1860, 114, 52. (d) Würtz, A. Justus Liebigs Ann. Chem. 1862, 121, 227.

2. Möller, F. Methoden der Organische Chemie (Houben-Weyl), 4th Edn; Müller, E., Ed.; Thieme Verlag: Stuttgart, 1957, Vol. 11/1, p 311-326.

3. Grayson, M. Ed. Kirk-Othmer Encycl. Chem. Technol. 1982, 17, 311.

4. Tritylated 2-aminoethanol was used many times for introduction of 2-aminoethoxy groups into target molecules, mainly oligonucleotides. It was prepared in the reaction of trityl, or trityl like, chloride or bromide with 2-aminoethanol. For example see: (a) Billimoria, J.D.; Owen, J.S.; Scott, G.H. Chem. Phys. Lipids 1974, 12, 327; Chem.Abstr. 1975, 83, 131086u. (b) Butskus, P.F.; Sabonene, P.Yu.; Lemeshene, D.B. Zh. Org. Khim. 1970, 6, 1984. (c) Kawakami, J.; Uesugi, S.; Ikehara, M.; Itoh, T.; Miura, K.; Ohtsuka, E. Nucleosides Nucleotides 1994, 13, 421; (d) Kormendy, K.; El-Sawy, M.M. Acta Chim. Acad. Sci. Hung. 1974, 83, 107. (e) Lammers, J.G.; van Boom, J.H. Recl. Trav. Chim. Pays-Bas 1979, 98, 243. (f) Maltese, M. J. Org. Chem. 2001, 66, 7615. (g) Perlman, N.; Livneh, M.; Albeck, A. Tetrahedron 2000, 56, 1505. (h) Tanaka, T.; Tamatsukuri, S.; Ikehara, M. Tetrahedron Lett. 1987, 28, 2611. (i) Tanaka, T.; Yamada, Y.; Ikehara, M. Chem. Pharm. Bull. 1988, 36; 1386. (j) Vinogradov, S.V.; Doan, T.L.; Helene, C. Tetrahedron Lett. 1995, 36, 2493. NTriphenylmethyl-2,2-dideuterioethanoloamine was prepared by reduction of tritylated glycine with LiAlD 4 . (k) Wang, J.J.-H.; Chan, K.K. J. Labelled Compd. Radiopharm. 1996, 38,105 . The reaction of tritylation of 2-aminoethanol was investigated in details by Butskus (ref. 4b) and Koermendy (Ref. 4d). There is only one example of tritylation of $\beta$ aminoalcohol other than 2-aminoethanol, described in. (1) Bol, K.M.; Liskamp, R.M.J. Tetrahedron Lett. 1991, 32, 5401. (m) Bol, K.M.; Liskamp, R.M.J. Tetrahedron 1992, 48, 6425.

5. Soroka, M.; Zygmunt, J. Synthesis 1988, 370.

6. In fact, we have found only one paper in which the reaction of 4-chlorophenylglycidyl ether with tritylamine was described, see: Desai, R.C. J. Org. Chem. 2001, 66, 4939. 
7. We have previously checked several solvents and found methanol to be superior over any other solvent. Isopropanol - a common solvent for epoxide ring opening reaction gave rather low yields of the desired product

8. For example: (a) Auge, J.; Leroy, F. Tetrahedron Lett. 1996, 37, 7715. (b) Browne, C.L.; Lutz, R.E. J. Org. Chem. 1952, 17, 1187. (c) Burfield, D.R., et al. J. Chem. Soc., Perkin Trans. 1 1977, 666. (d) Chapman, N.B.; Triggle, D.J. J. Chem. Soc. 1963, 1385. (e) Chini, M.; Crotti, P.; Macchia, F. J. Org. Chem. 1991, 56, 5939. (f) Chini, M.; Crotti, P.; Macchia, F. Tetrahedron Lett. 1990, 31, 4661. (g) Gabriel, S.; Stelzner, R. Chem. Ber. 1896, 29, 2381. (h) Padwa, A.; Chen, Y.; Chiacchio, U.; Dent, W. Tetrahedron 1985, 41, 3529. (i) Potekhin, A.A.; Safronov, A.O. Zh. Org. Khim. 1981, 17, 379. (j) Rampalli, S.; Chaudhari, S.S.; Akamanchi, K.G. Synthesis 2000, 78. (k) Remuzon, P.; Soumeillant, M.; Dussy, C.; Bouzard, D. Tetrahedron 1997, 53, 17711. (l) Stolberg, M.A.; O’Neill, J.J.; WagnerJauregg, T. J. Amer. Chem. Soc. 1953, 75, 5045. (m) Weghe, P. Van de; Collin, J. Tetrahedron Lett. 1995, 36, 1649. (n) Wilson, W. J. Chem. Soc. 1952, 3524. (o) Yamada, J.; Yumoto, M.; Yamamoto, Y. Tetrahedron Lett. 1989, 30, 4255. (p) Yamamoto, Y.; Asao, N.; Meguro, M.; Tsukada, N.; Nemoto, H.; Sadayori, N.; Wilson, J.G.; Nakamura, H. J. Chem. Soc. Chem. Commun. 1993, 15, 1201.

9. For example: (a) Acheson, R.M.; Bite, M.G.; Kemp, J.E.G. J. Med. Chem. 1981, 24, 1300. (b) Filipek, B.; Sapa, J.; Malawska, B.; Kulig, K.; Antkiewicz-Michaluk, L. Arch. Pharm. (Weinheim) 1997, 330, 225. (c) Hoefle, M.L.; Blouin, L.T.; Fleming, R.W.; Hastings, S.; Hinkley, J.M.; Mertz, T.E.; Steffe, T.J.; Stratton, C.S.; Werbel, R.M. J. Med. Chem. 1991, 34, 7. (d) Protiva, M.; Sustr, M.; Borovička, M. Chem. Listy 1951, 45, 43; Chem. Abstr. 1951, 45, 9010e.

10. The crude product contains some polymeric fraction which could be removed by recrystallization from dioxane/water.

11. Because its steric hindrance and high symmetry, the trityl group is dominating over the rest of molecule. This effect increases the melting points of all tritylated products and makes crystallization easy, so the trityl group is really the "crystal maker".

12. The regioselectivity of the ring opening of propylene and butylene oxide is usually ignored in published papers. Authors believed that ring opening is giving always "beta" products.

13. It is worth to note that even before introduction of modern NMR instruments into organic labs, it was possible to determine the ratio of ring opening products just by recrystallization of the N-benzoyl derivatives of both aminoalcohols, for example, in the reaction of styrene oxide and ammonia. See: Castro, A.J.; Brain, D.K.; Fisher, H.D.; Fuller, R.K. J. Org. Chem. 1954, 19, 1444.

14. The 1-phenyl-2-(triphenylmethylamino)ethanol was hydrolyzed 15 minutes in $2 \mathrm{M}$ hydrochloric acid, since concentrated hydrochloric acid gave also about 30\% (molar) of the 2-chloro-2-phenylethyl(triphenylmethyl)amine which could be converted to the 2-amino-1phenylethanol by increasing the time of hydrolysis. This chemistry, related to hydramine fission, is under investigation.

15. Gaertner, V.R. J. Org. Chem. 1967, 32, 2972. 